Journal of Earthquake and Tsunami

Vol. 13, Nos. 5 \& 6 (2019) 1941006 (15 pages)

(C) The Author(s)

DOI: $10.1142 /$ S1793431119410069

\title{
Boulder Pickup by Tsunami Surge
}

\author{
Samuel Harry*, Margaret Exton ${ }^{\dagger}$ and Harry Yeh* \\ Civil and Construction Engineering \\ Oregon State University \\ Corvallis, OR 97331, USA \\ *harrys@oregonstate.edu \\ †extonm@oregonstate.edu \\ †harry@oregonstate.edu \\ Received 14 January 2019 \\ Accepted 7 June 2019 \\ Published 23 August 2019
}

\begin{abstract}
Study of boulder transport by tsunamis is challenging because boulder size, shape, and composition vary greatly; furthermore, flow conditions, topography, and initial conditions are generally unknown. To investigate the mechanism of boulder pickup, experiments of tsunamilike flow past spherical boulders partially buried in a sediment bed are conducted. The experiments are performed in a large centrifuge facility to reduce scale effects and the corresponding dynamic similitude is discussed. The traditional approach to determine boulder pickup is adapted for the case of a half-buried spherical boulder. The adapted model predicts that the boulders are transported, but does not accurately predict the timing of pick up. To investigate the difference in pickup timing, two physical phenomena are discussed: pore-water-pressure dissipation in the soil, and the impact of the free-surface flow on hydrodynamic forces. For a spherical shaped boulder, vertical forces (i.e. buoyant and lift forces) are critical for the initiation of boulder pickup. It was found that spherical boulders that are three-quarter buried in the soil are not transported, even when exposed to flow conditions that would otherwise predict transport.
\end{abstract}

Keywords: Tsunami; boulders; centrifuge.

\section{Introduction}

There is much reported evidence of boulder transport and deposition caused by tsunamis. Boulders have been kept as monuments to a tsunami event and can be found in many places in Japan, serving to remind people of the rare but high-impact natural disaster. Figure 1 exhibits clear evidence of tsunami-transported boulders found in survey after the 1992 Flores Tsunami. Also, the beach rock shown in Fig. 1

\$Corresponding author.

This is an Open Access article published by World Scientific Publishing Company. It is distributed under the terms of the Creative Commons Attribution 4.0 (CC BY) License which permits use, distribution and reproduction in any medium, provided the original work is properly cited. 


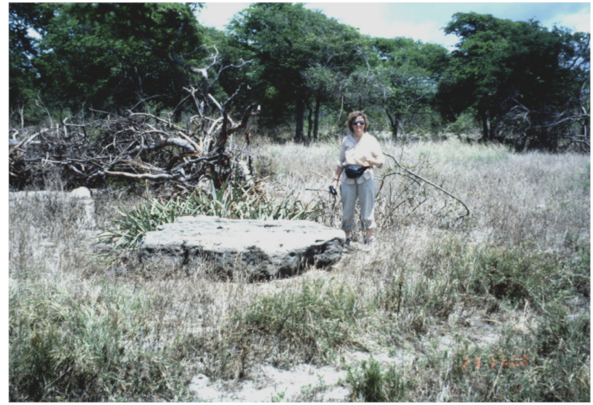

(a)

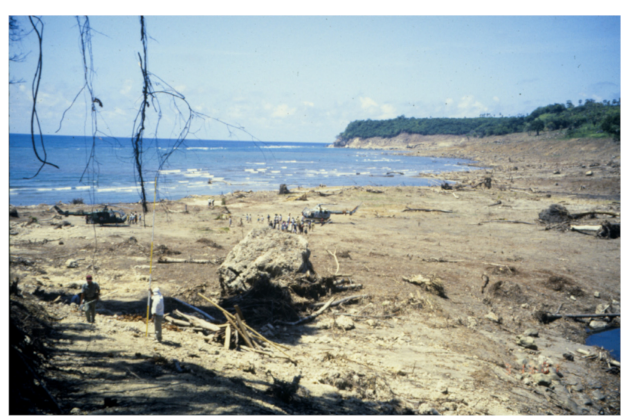

(b)

Fig. 1. Photographs of boulders found in the field. (a) A beach rock transport by the 1992 Nicaragua Tsunami. The rock was deposited on the fresh vegetation, proving that it was indeed transported by the tsunami. (b) A scene of Trubean, Flores, Indonesia, immediately after the 1992 Flores Tsunami. A large boulder is observed, as well as many other boulders in the far site along the beach. Those boulders are thought to be bulks of corrals.

was found on fresh vegetation immediately after the 1992 Nicaragua Tsunami, proving that the boulder was indeed transported by the tsunami. Boulder composition, location, and/or other site specific details can provide valuable information about historical tsunami events, and therefore contribute to the record of the frequency and magnitude of significant tsunami events. Boulder transport processes are complex due to transient, highly turbulent, free-surface flows directly interacting with a lump of solid. In addition, the size and composition of boulders vary, as does topography. Hence, to understand boulder transport mechanisms, it is important to approach this problem in a controlled laboratory environment.

Existing boulder transport models range from one-dimensional analysis to numerical models that couple hydrodynamics and boulder motion. In the context of tsunamis, Noji et al. [1993] proposed a boulder transport model based on a combination of drag and inertia forces acting on the object; this approach is similar to the Morison equation often used to estimate the wave force exerted on vertical piles (see for example Dean and Dalrymple [1991]). The same approach was used by Petroff et al. [2001] and Imamura et al. [2008] for their analyses of laboratory, numerical, and field data analyses. The initiation of boulder transport is determined by the force balance of the hydrodynamic action with the resistive and restoring forces or the moment balance of the hydrodynamic action for the rolling failure. The lift force was considered by Nott [2003], Nandasena et al. [2011], Nandasena and Tanaka [2013], and Kennedy et al. [2016]. Miscellaneous forces may also be accounted for, such as the force needed to dislodge a boulder that is partially attached to a rock face [Noormets et al., 2004]. Models of hydrodynamic forces are experimentally calibrated by comparing hydrodynamic conditions to boulder motion [Noji et al., 1993; Imamura et al., 2008; Nandasena and Tanaka, 2013]. Field scale comparisons of hydrodynamic conditions to boulder motion are achieved by installing accelerometers into boulders to track the time-evolution and mode of transport [Stephenson and Abazović, 2016]. 
Hydrodynamic conditions for tsunami flows have speeds roughly in the order of five meters per second and depths in the order of ten meters. For example, video footage of the 2011 Heisei Tsunami (also known as the Tohoku-Oki Tsunami) taken in Onagawa, Japan, indicates that typical flow speeds are approximately $6.3 \mathrm{~m} / \mathrm{s}$ for runup and $7.5 \mathrm{~m} / \mathrm{s}$ for drawdown with a flow depth of about $4.0 \mathrm{~m}$ [Koshimura and Hayashi, 2012]. The speed of the leading front of the runup surge measured from the Sendai Plain (also reported by Koshimura and Hayashi [2012]) was approximately 6 to $8 \mathrm{~m} / \mathrm{s}$ with the flow depth of 1 to $2 \mathrm{~m}$. Replicating such flows at full scale in a laboratory is difficult, so reduced scale experiments must be conducted.

Investigating boulder transport is complex for many reasons: boulders vary greatly in size and shape, and are composed of a variety of materials such as rock or coral as shown in Fig. 1, pre-transport conditions for the boulder are generally unknown including burial depth, the surrounding soil composition, and topographic variation, which affects both the hydrodynamic conditions and the resistive forces applied to the boulder.

Previous experiments measure changes in boulder position at particular stages of the flow, e.g. Petroff et al. [2003] and Imamura et al. [2008], or utilize imaging to track boulder translation with the flow conditions, e.g. Luccio et al. [1998], Nandasena and Tanaka [2013], and Liu et al. [2014]. Such experiments excel in allowing large-scale measurements of motion, but small incipient motions may be obscured by the water surface. Accelerometers may be used to precisely identify the timing and mode of incipient motion in field scale experiments, as shown in Stephenson and Abazović [2016], however installing such accelerometers in small-scale model boulders is difficult. Thus, in order to identify incipient boulder motion when imaging is obscured by the aerated and sediment laden flow, we utilize a proximity sensor as will be discussed in Sec. 3. Previous experiments have been conducted on rigid and planar beds. Such experiments account for a sloping bottom, but neglect the influence of the underlying sediment bed. Here, we conduct the experiments incorporating the impact of an underlying sediment bed. Furthermore, we focus the study on the exploration of the mechanisms of incipient boulder motion due to a tsunami-like flow.

\section{Scaling}

To reduce scale effects, which are often substantial and unavoidable, laboratory experiments are conducted in a large centrifuge facility. The centrifugal force is used to control the body force in the model. The following discussion considers a generic increase in body force by a factor of $N$, induced by the centripetal acceleration. Dynamic similitudes between model and prototype for both the fluid and soil domains are discussed.

A fluid domain is governed by the Navier-Stokes equation, which is written in dimensionless form as

$$
S \frac{\partial \vec{u}}{\partial t}+\vec{u} \cdot \nabla \vec{u}=-\frac{1}{E} \nabla p+\frac{1}{R} \nabla^{2} \vec{u}+\frac{1}{F^{2}} \vec{k}
$$


where $\vec{u}$ is the fluid velocity, $p$ is the fluid pressure, $\vec{k}$ is the unit vector pointing in the direction of body force, $\nabla$ is the del operator, and the arrow bar represents vectors. The dimensionless parameters appearing in the Navier-Stokes equation (2.1) are:

$$
\begin{array}{r}
\text { Strouhal number: } S=\frac{L_{0}}{u_{0} t_{0}} ; \quad \text { Euler number: } E=\frac{\rho_{0} u_{0}^{2}}{p_{0}} ; \\
\text { Reynolds number: } R=\frac{\rho_{0} u_{0} L_{0}}{\mu_{0}} ; \quad \text { Froude number: } F=\frac{u_{0}}{\sqrt{g_{0} L_{0}}} ;
\end{array}
$$

where $L_{0}, t_{0}, u_{0}$, and $p_{0}$ are representative scales of length, time, speed, and pressure, respectively, and $\rho_{0}, \mu_{0}$, and $g_{0}$ are the fluid density, viscosity, and body force per unit mass, respectively. Since the body force in the model is increased by a factor of $N$, the length and time scales are reduced by a factor of $N$ to observe the Froude law. Consequently, while the Strouhal and Euler numbers match between prototype and model, the Reynolds number mismatches by $N$. In other words, the model fluid must have $1 / N$ the viscosity of the prototype fluid to match the Reynolds number of the prototype.

For the sediment domain, consider the dynamics being governed by the scaled Terzaghi equation as a simplified model:

$$
\frac{\partial p_{e}}{\partial t}=T \nabla^{2} p_{e}, \quad T=\frac{\kappa t_{0}}{\mu_{0} L_{0}^{2}}\left(\frac{1+e_{0}}{a_{v}}\right)=\frac{\kappa t_{0}}{\mu_{0} L_{0}^{2}}\left(\frac{E_{v}}{1-\phi}\right),
$$

where $p_{e}$ is the excess pore water pressure, $T$ is the Terzhagi number (also known as the time factor of consolidation), $\kappa$ is the intrinsic permeability, $e_{0}$ is the void ratio of the soil, and $a_{v}$ is the coefficient of compressibility. Equivalently, we can express the Terzhagi number $T$ in terms of porosity $\phi$ and the bulk modulus of elasticity of the fluid $E_{v}$. When the particle size is not scaled, the soil properties match between prototype and model. Consequently, the Terzaghi number mismatches by $N$. One way to account for this is to scale the viscosity of the model pore fluid by $N$ times more than the prototype pore fluid to match the pore-pressure behavior of the prototype. Note that this requirement is a contradiction to that needed to satisfy the Reynolds number similitude.

The boundary between the fluid and soil domain can be characterized by the Shields parameter

$$
\Theta=\frac{\tau_{0}}{\rho_{0} g_{0} d_{0}\left(G_{s}-1\right)},
$$

where $G_{s}$ is the specific gravity of the sediment particles, $d_{0}$ is the diameter length scale of the sediment grains, and $\tau_{0}$ is the bed shear stress. In the experiments described herein, the flow is fully turbulent, so the bed shear stress can be described by $\tau_{0} \propto \rho u^{2}$. The sediment particle size is usually not scaled in traditional geotechnical experiments so as to match the soil properties between model and prototype. Consequently, the Shields parameter mismatches by $N$. In other words, the soil bed 
Table 1. Scaling mismatch for non-dimensional parameters and physical variables as a ratio of prototype to model. The $1 \mathrm{~g}$ and $\mathrm{Ng}$ models are scaled according to Froude law where the fluids and sediments are identical between model and prototype. Note that Froude-law scaling is not common for geotechnical experiments studying phenomenon characterized by the Terzhagi number.

\begin{tabular}{lcccccccccc}
\hline & $S$ & $E$ & $R$ & $F$ & $T$ & $\Theta$ & $L_{0}$ & $t_{0}$ & $u_{0}$ & $p_{0}$ \\
\hline $1 g$ Model & 1 & 1 & $N^{\frac{3}{2}}$ & 1 & $1 / N^{\frac{5}{2}}$ & $N$ & $N$ & $\sqrt{N}$ & $\sqrt{N}$ & $N$ \\
$N g$ Model & 1 & 1 & $N$ & 1 & $1 / N$ & $N$ & $N$ & $N$ & 1 & 1 \\
\hline
\end{tabular}

is more stable for the model and does not match the sediment transport behavior of the prototype.

While the experiments in the centrifuge environment cannot achieve perfect dynamic similitudes, the scale effects are much reduced from those associated with experiments under the normal gravitational environment. With the use of water in the experiments, the mismatch in Reynolds number is reduced by $\sqrt{N}$ in the centrifuge; hence the flow in the model can approach the regime of "fully" turbulent hopefully so that the influence of the Reynolds number may become unimportant. Furthermore, in the centrifuge, both flow velocities and pressures can be reproduced identical to the magnitudes of the prototype. Considering the conditions where the fluids and sediments are identical between model and prototype, the scaling for each parameter in commonly performed experiments $(1 g)$ and centrifuge experiments $(\mathrm{Ng})$ are shown in Table 1.

\section{Experiments and Measurements}

Experiments were conducted in a flow tank designed for use in a centrifuge as shown in Fig. 2. For the present study, the flow tank is operated on the centrifuge at $40 \mathrm{~g}$ centripetal acceleration (i.e. $N=40$ ), and we consider the geometric scale of the model to be $1 / 40$ of the prototype. Dam-break flow is generated to simulate tsunami inundation as illustrated in Figs. 2(b) and 2(c). The soil model was prepared with Ottawa F65 silica sand: the mean grain diameter is $0.21 \mathrm{~mm}$ and the specific gravity of the grains is 2.65. The soil was dropped uniformly from a predetermined height to achieve a porosity of 0.38 . To achieve the fully saturated state, we first create a near vacuum condition in the soil pore space and inject more soluble carbon dioxide gas. This process is repeated and the soil model is brought again to a near vacuum condition. Then, prepared de-aired water is carefully introduced in the soil specimen. After saturation, the vacuum is released to the atmospheric pressure state.

Three sizes of spherical-shaped model boulders are used for the experiments: those are $12 \mathrm{~mm}, 16 \mathrm{~mm}, 25 \mathrm{~mm}$ in diameter. The boulders are made of silica glass which has a specific gravity of 2.6 (close to that of the soil grains). The initial pickup motion is detected by a 'proximity sensor' as shown in Fig. 3(a). The proximity sensor is buried in the soil directly beneath the boulder, such that the magnet initially contacts the sensor, to measure the strength of the magnetic field, utilizing the Hall 
(b)

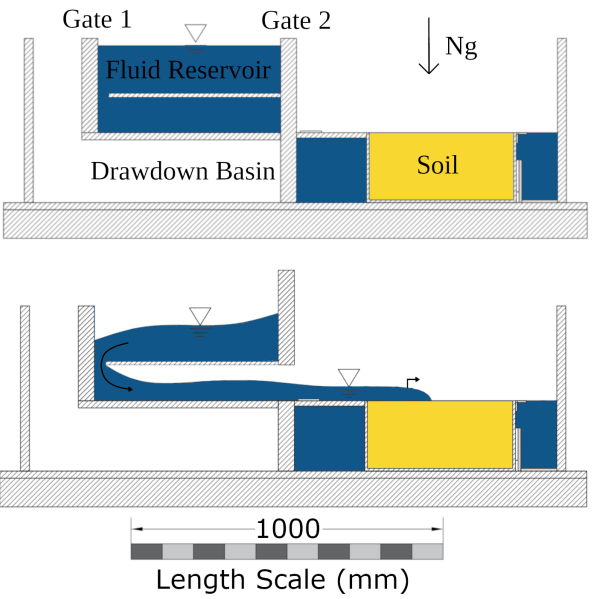

Fig. 2. Laboratory apparatus and operation. (a) A schematic sketch of centrifuge operation, not to scale. Note that the centrifuge apparatus is 9 meters in radius. (b) Initial condition for the model. The reservoir was filled with water, the soil model was saturated, and the compartment of the flow tank surrounding the soil model was filled with water until flush with the top of the soil model. The centrifuge begins to spin and swings the model rotated clockwise by $90^{\circ}$ at the end of the centrifuge arm as shown in (a). (c) A sketch of water flow after opening the gate. The water flows downward though the opening near gate 1 , then across the floor of the reservoir, where the flow vanes guide and regulate the flow. This design restricts the flow, reducing the speed and impact of the Coriolis force on falling fluids.

(a)

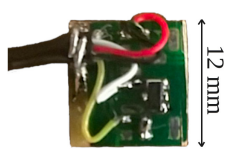

(b)
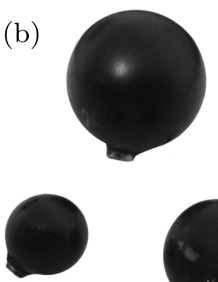

(c)

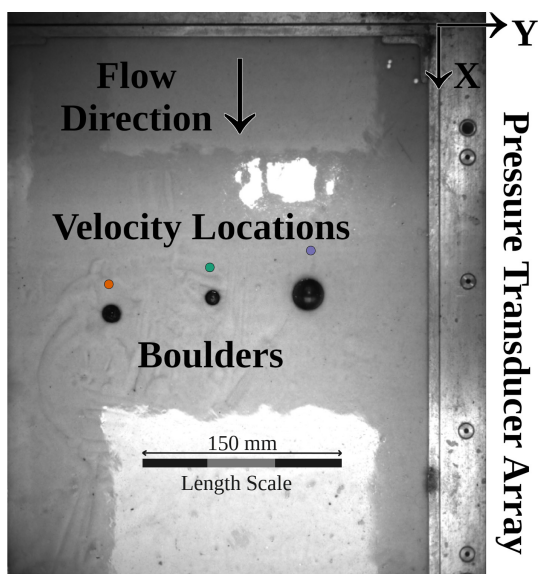

Fig. 3. Experimental setup. (a) Photograph of proximity sensor. The sensor consists of a Hall effect sensor, capacitor, and wire leads soldered to a printed circuit board. (b) Photograph of model boulders: $12 \mathrm{~mm}, 16 \mathrm{~mm}$, and $25 \mathrm{~mm}$ in diameter. The boulders are glass spheres, painted black, and affixed with a small magnet. (c) Plan view of the soil model container during Trial One. Primary direction of flow comes from the top of the photo, the origin is taken at the top right corner of the soil container, the flow velocity is sampled at the colored dots slightly upstream of each boulder as shown, and the pressure transducer array is located on the right side of the container. The velocity sampling locations are taken, in millimeters as $(X, Y)$ pairs from left to right, at $(201,-256),(188,-178)$, and $(175,-100)$. 
effect, of the small magnet affixed to the boulder (Fig. 3(b)). The effective range of the proximity sensor is dependent on the size and strength of the affixed magnet. With the chosen magnet, the proximity sensor detects motion within about four millimeters of the sensor. Since the strength of magnetic field measured by the proximity sensor depends on the distance and orientation of the magnet relative to the sensor, distance cannot be directly measured by the sensor. A change in magnetic field only indicates motion, either rotation or translation, of the boulder relative to the proximity sensor. However, the proximity sensor provides a reliable and precise threshold of movement when the boulder is visually obscured by the highly aerated flow at the front of the tsunami surge.

Two trials were conducted in this experimental program. Due to the resourceintensive nature of conducting centrifuge experiments, trials were not repeated. In Trial One, the $12 \mathrm{~mm}, 16 \mathrm{~mm}$, and $25 \mathrm{~mm}$ spherical model boulders were half-buried in the soil directly above the proximity sensors, such that the affixed magnet made contact with the sensor. Note that the $12 \mathrm{~mm}, 16 \mathrm{~mm}$, and $25 \mathrm{~mm}$ diameter model boulders correspond to $0.48 \mathrm{~m}, 0.64 \mathrm{~m}$, and $1.0 \mathrm{~m}$ diameter boulders in prototype scale, respectively. The boulders were placed in the soil as shown in Fig. 3(c). In Trial Two, proximity sensors were not placed, and three $16 \mathrm{~mm}$ boulders (corresponding to $0.64 \mathrm{~m}$ diameter boulders in prototype scale) were buried in the saturated soil with one quarter, one half, and three quarters burial depth. The boulders were placed in the soil with a similar layout to Trial One.

Flow depth is estimated with pressure transducers placed at the bed elevation in a linear array in the $X$-direction, parallel to the primary direction of flow (see Fig. 3(c)). The water depth at the boulders is estimated with the pressure transducer nearest the boulder locations. Assuming the vertical accelerations of the fluid are small, the water depths are estimated by a hydrostatic condition as shown in Fig. 4(a). Except the very small leading edge of the surge $(t<0.05 \mathrm{~s})$ where splash up affects the waveform, this assumption is validated by optical observations made with a high-speed video with a horizontal perspective. The water surface profile in Fig. 4(a) shows the formation of a "head" followed by the steady increase of the depth. The formation is in agreement with the model proposed by Whitham [1955] who assumed that the leading tip of the surge must be treated as analogous to the boundary layer (i.e. viscous effects are significant). The flow behind the head increases steadily with depth while the flow speed appears nearly constant. Hence, the leading tip of the surge moves like a solid body.

Flow velocity is obtained using two-frame motion estimation using a high speed video camera whose top down perspective is shown in Fig. 3(c). Using the aeration in the leading portion of the surge, an optical flow algorithm combined with images of the flow is capable of estimating the flow velocity [Bung and Valero, 2016]. Utilizing high-speed imaging and the optical flow algorithm developed by Farnebäck [2003], flow velocity is evaluated at every point over the soil model. The patterns of light created by the aerated flow allow the optical flow to be estimated accurately. The accuracy of this method is validated by comparing the results of two-frame motion 


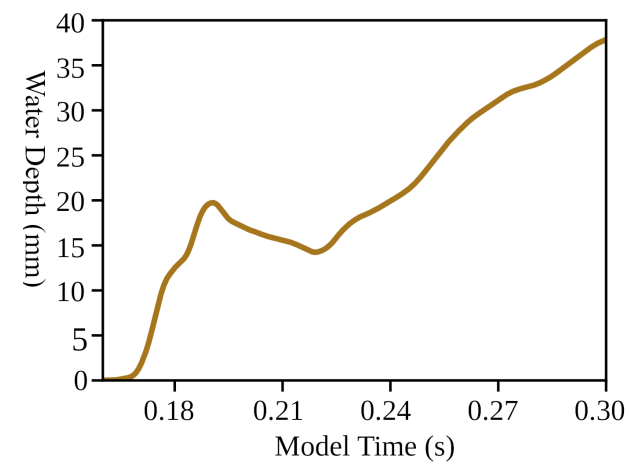

(a)

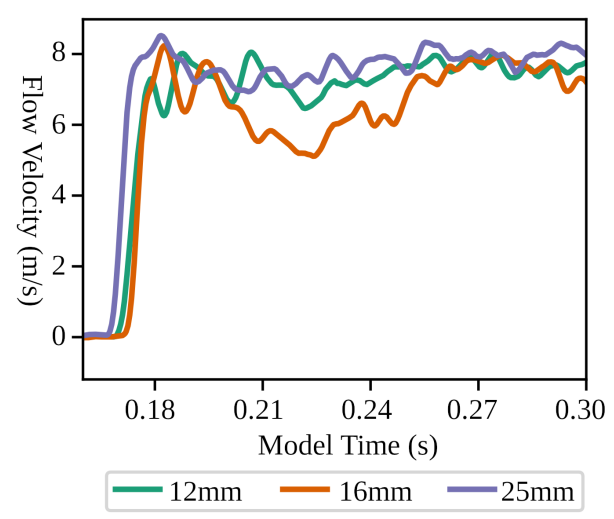

(b)

Fig. 4. Flow characteristics for Trial One. (a) Estimated flow depth at the pressure transducer nearest the boulders. (b) Estimated flow velocities in the $X$-direction at the locations corresponding to the sampling locations slightly upstream of the boulders shown in Fig. 3(c).

estimation to tracking the position of flow tracers distributed in the flow. Flow tracers show that the two-frame motion estimation tends to underpredict the flow velocity by $0.18 \mathrm{~m} / \mathrm{s}$ on average when the flow is highly aerated near the front of the surge, which is small in comparison with the leading flow speed $(\approx 7.5 \mathrm{~m} / \mathrm{s})$. The velocities near the model boulders are shown in Fig. 4(b). Non-uniform flow velocities in the $Y$-direction are observed, consequently flow depth is expected to be nonuniform in the $Y$-direction, but the variation in flow depth cannot be quantified with the foregoing experimental setup.

A comparison of proximity sensor signals and visual tracking of boulder motion is shown in Fig. 5. The proximity sensors are proficient in detecting both a precise timing of initiation of movement as well as the duration of the dislodgement process. The dislodgement process is about $0.03 \mathrm{~s}$ for the $12 \mathrm{~mm}$ boulder, about $0.02 \mathrm{~s}$ for the $25 \mathrm{~mm}$ boulder, and about $0.07 \mathrm{~s}$ for the $16 \mathrm{~mm}$ boulder. The large dislodgement duration of the $16 \mathrm{~mm}$ boulder compared to the $12 \mathrm{~mm}$ and $25 \mathrm{~mm}$ boulders is likely caused by non-uniform flow conditions in the model, as indicated by Fig. 4(b). The sudden (discontinuous) change in magnetic field measured by the $25 \mathrm{~mm}$ boulder is puzzling and is most likely caused by a sensor artifact, rather than the actual boulder motion. Nonetheless, this demonstrates the applicability of the sensing method, all in all.

For the flow conditions shown in Fig. 4, the non-dimensional parameters to describe the flow about the model boulders can be found in Table 2. Everything is realistically simulated for a typical tsunami inundation in the centrifuge model, except the Reynolds number mismatches by $N(=40)$. Note that an isolated smooth sphere transitions from laminar separated flow to turbulent separated flow at around $R_{\text {sphere }} \sim 5 \times 10^{5}$; hence, the scale effect in terms of Reynolds number mismatch is present, though its extent is unknown. 


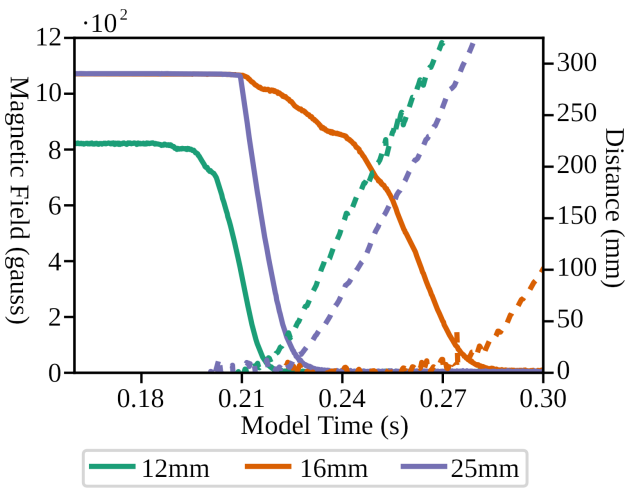

(a)

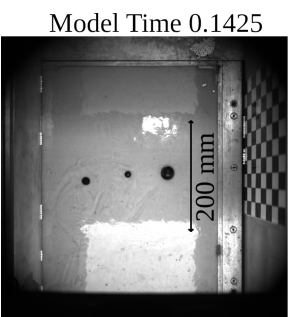

Model Time 0.2325

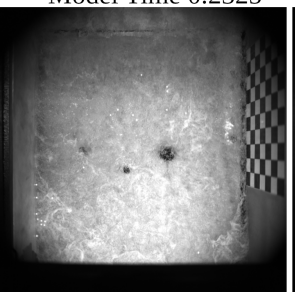

(b)
Model Time 0.2225

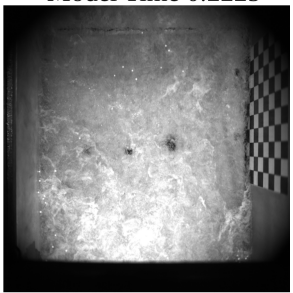

Model Time 0.2825

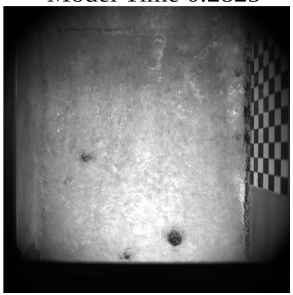

Fig. 5. Comparison of proximity sensor and visual tracking results in Trial One. In (a), the solid lines represent magnetic field measured by the proximity sensor, the dashed lines are the distance traveled by the boulder based on the image analysis of the high-speed video data, and colors correspond to the $12 \mathrm{~mm}$, $16 \mathrm{~mm}$, and $25 \mathrm{~mm}$ boulders as indicated in the legend. The visual tracking is made by estimating the centroid of the boulders in the image. (b) Visuals of boulder motion.

Table 2. Non-dimensional parameters for the flow about the boulders for the $40 \mathrm{~g}$ model and corresponding prototype.

\begin{tabular}{lccccc}
\hline & $R_{\text {boulder }}$ & $F$ & $L_{\text {boulder }}$ & $h_{0}$ & $u_{0}$ \\
\hline Model & $9.0 \times 10^{4}-1.87 \times 10^{5}$ & $2-3$ & $12 \mathrm{~mm}-25 \mathrm{~mm}$ & $15 \mathrm{~mm}-35 \mathrm{~mm}$ & $7.5 \mathrm{~m} / \mathrm{s}$ \\
Prototype & $3.6 \times 10^{6}-7.5 \times 10^{6}$ & $2-3$ & $0.48 \mathrm{~m}-1.0 \mathrm{~m}$ & $0.6 \mathrm{~m}-1.4 \mathrm{~m}$ & $7.5 \mathrm{~m} / \mathrm{s}$ \\
\hline
\end{tabular}

\section{Analysis and Discussion}

Considering the previous work [e.g. Noji et al., 1993; Petroff et al., 2001; Nott, 2003; Imamura et al., 2008; Nandasena et al., 2011], fluid forces on a boulder are modeled by a combination of drag, inertial, and lift forces together with the stabilizing body force. All of the previous works considered the boulder shape to be cuboid. Here, we first present the same traditional force decomposition but for a spherical shaped object with the radius $a$ buried in the soil by one half diameter

$$
\begin{gathered}
F_{\text {drag }}=\frac{1}{2} \rho C_{D} A_{D} U^{2}=\frac{\pi}{8} \rho a^{2} U^{2}, \\
F_{\text {inertial }}=\rho C_{I} V_{f} \frac{\partial U}{\partial t}=\pi \rho a^{3} \frac{\partial U}{\partial t}, \\
F_{\text {lift }}=\frac{1}{2} \rho C_{L} A_{L} U^{2}=\rho a^{2} U^{2},
\end{gathered}
$$




$$
F_{\text {body }}=\left(\rho_{s}-\rho\right) g V=\frac{4 \pi}{3} S_{g} \rho a^{3},
$$

where $C_{D}, C_{I}$, and $C_{L}$ are the drag, inertial, and lift coefficients, respectively; $A_{D}$ and $A_{L}$ are the area of the object exposed to the flow projected to the plane normal and tangential to the ambient flow direction, respectively; $V_{f}$ is the volume of the object exposed to the flow; $V$ is the total volume of the object; $U$ and $\partial U / \partial t$ are the ambient flow velocity and its temporal derivative, respectively; $\rho$ and $\rho_{s}$ are the density of the fluid and solid, respectively; $S_{g}$ is the specific gravity of the solid, $\left(\rho_{s}-\rho\right) / \rho$. We take the value of the lift and inertial coefficients from the analytical solutions of potential flow past a sphere [Lamb, 1932], namely $C_{L}=\frac{2}{\pi}$ and $C_{I}=\frac{3}{2}$. For these values, we consider a no flux condition on the soil bed (the $X-Y$ plane); hence we assume the flow is stagnant below the soil bed to compute the lift force upward on the boulder. Such an idealized model is used in lack of experimental values. The drag coefficient is chosen as $C_{D}=0.5$ from experiments of flow around a sphere (see for example Prandtl and Tietjens [1934]). The area of lift and drag were taken as $\pi a^{2}$ and $\frac{1}{2} \pi a^{2}$, respectively, for a half buried sphere with the radius $a$.

In Fig. 6, the estimated fluid forces are compared for Trial One together with the response of the proximity sensor that monitors detachment of the boulder. Recall that in Trial One, the $12 \mathrm{~mm}, 16 \mathrm{~mm}$, and $25 \mathrm{~mm}$ spherical boulders were buried to one half diameter in the soil. In all cases, the inertial forces quickly subside after the initial impact, then become small before any movement of the boulders. This clearly indicates that for half-buried spherical-shaped boulders, the inertial forces are unimportant to induce the pickup movement, despite being on the same order of magnitude as the subsequent drag and lift forces.

The lift and drag forces are assumed to act at the geometric center of $A_{L}$ and $A_{D}$. Taking the ratio of the moment generated by the drag force to the lift force

$$
\frac{M_{\mathrm{drag}}}{M_{\mathrm{lift}}}=\frac{\frac{4 a}{3 \pi} F_{\mathrm{drag}}}{a F_{\text {lift }}}=\frac{4 C_{D} A_{D}}{3 \pi C_{L} A_{L}}=\frac{1}{6},
$$

for the half buried sphere and coefficients chosen earlier. This indicates that the drag force does not significantly contribute to overturning the boulder, although such a conclusion is dependent on the values of coefficients.

The net upward thrust is determined by the difference of lift force (4.3) and buoyant weight (4.4), and is presented in Fig. 6(d) for the flow conditions of Trial One (see Fig, 4). It is cautioned that the estimated lift force by (4.3) is for fully submerged steady flows, which is not exactly the case here: the surging flow is shallow, turbulent and highly transient. The net upward thrust shown in Fig. 6(d) predicts that the boulders should move before any measured movement by the proximity sensors. The $0.014 \mathrm{~s}$ difference between the occurrence of the net upward force, and the measured movement for the $12 \mathrm{~mm}$ boulder is possibly attributed to sampling location, as the velocities are sampled slightly upstream of the boulder. The delay caused by sampling location is estimated by noting that the velocities are sampled about $50 \mathrm{~mm}$ upstream of the boulder's center, that the surge front 

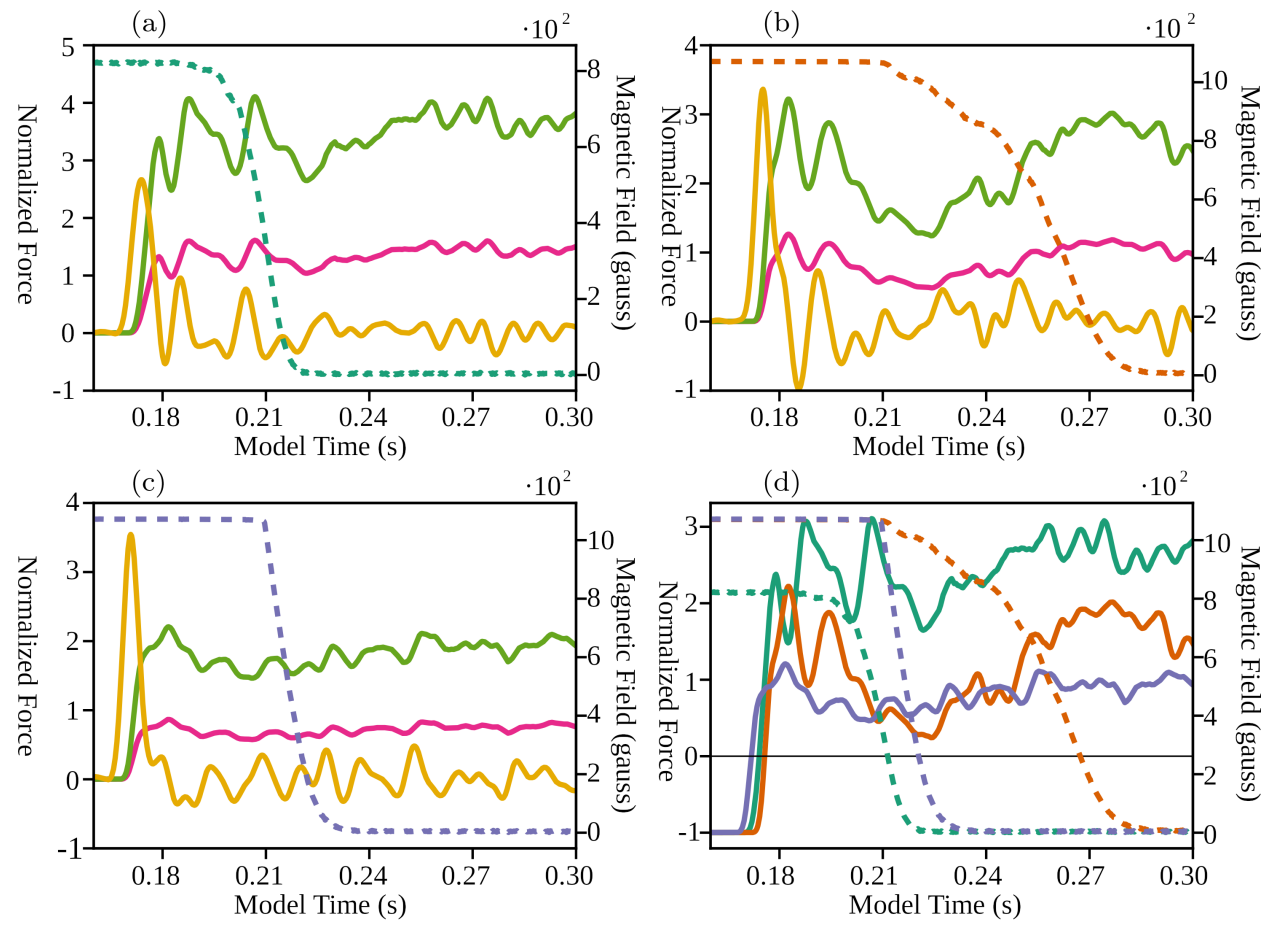

- drag lift $=$ inertial

$12 \mathrm{~mm}$

$16 \mathrm{~mm}$

$25 \mathrm{~mm}$

Fig. 6. Comparison of estimated hydrodynamic forces and proximity sensor results. Forces are normalized by the buoyant weight of the boulder in the model. (a) The $12 \mathrm{~mm}$ boulder: colored solid lines correspond to the estimated drag, lift, and inertial forces as indicated in the legend, and the dashed line corresponds to the proximity sensor response. (b) The $16 \mathrm{~mm}$ boulder: the presented solid lines are color coded the same as in (a), and the dashed line corresponds to the proximity sensor response. (c) The $25 \mathrm{~mm}$ boulder: the presented solid lines are color coded the same as in (a), and the dashed line corresponds to the proximity sensor response. (d) Net upward thrust is shown with solid lines, proximity sensor results are shown with dashed lines. Colors correspond to the $12 \mathrm{~mm}, 16 \mathrm{~mm}$, and $25 \mathrm{~mm}$ boulders as indicated in the legend.

propagates at about $8 \mathrm{~m} / \mathrm{s}$, and that the surge front completely submerges the boulders after propagating about $100 \mathrm{~mm}$ beyond the sampling point, or within $0.014 \mathrm{~s}$. Nonetheless, the $0.036 \mathrm{~s}$ and $0.038 \mathrm{~s}$ difference for the $16 \mathrm{~mm}$ and $25 \mathrm{~mm}$ boulders, respectively, are too large to be attributed to velocity sampling locations.

Although the temporal discrepancy between the estimated and the measured boulder movement may not be significant on the field scale when there are many unknowns, under laboratory conditions this discrepancy suggests that there are significant physical factors preventing the boulders from moving when they are first submerged by the flow. Two potential physical factors are considered: (1) pore water pressure effect in the soil applying a stabilizing force on the boulder, and (2) flow depth preventing full development of the lift force. 


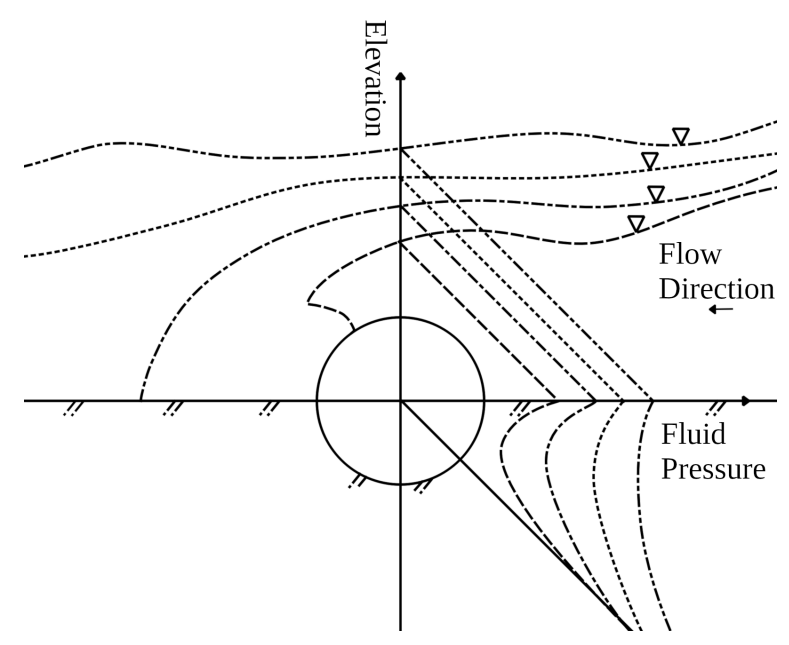

Fig. 7. A qualitative illustration of pore pressure behavior during inundation.

To investigate the influence of soil on the boulder transport process, soil domains governed by Terzaghi's equation are considered. As shown in Yeh and Mason [2014], due to the transient nature of the flow, the pore-water pressure gradient in the soil during tsunami runup points upwards, causing a downward stabilizing force on the sediment bed. This force would stabilize any object buried in the soil. The stabilizing force would be transient, and be strongest at the leading edge of the surge, causing a delay in the transport of the boulder, but not preventing it entirely. An illustrative sketch shown in Fig. 7 exhibits anticipated representative pore-water pressure variations during the surge-front passage assuming hydrostatic pressure in the fluid domain. Note that at the very tip of the surge front, the pressure could be far from hydrostatic due to the strong vertical accelerations. Pore-water pressure beneath the partially buried sphere are not known, unfortunately; hence the influence of diffusive pore-water pressure is not verified.

Boulders with heights protruding from the soil comparable to the flow depth may have the surrounding flow affected by the water surface. The lift force estimated in (4.3) is for steady flows and neglects the flow depth: the coefficient $C_{L}=\frac{2}{\pi}$ is from a mathematical model of potential flow about a half buried sphere in an infinite fluid domain [Lamb, 1932]. To illustrate this effect, let us consider the steady potential flow. Under this circumstance, a free surface is present where the streamlines have constant velocity and have constant elevation some distance away from the boulder. If we consider a surface above the location of the boulder at an elevation three times the sphere radius, then streamlines have nearly constant elevation and the percent difference of the local flow velocity from the ambient flow velocity is less than $1.85 \%$ along the streamline, approximately satisfying the free surface condition. This suggests that the flow depth must be three times the boulder radius or greater for the lift force to be independent of flow depth. With this simplified condition, we assume that 
the flow must be $18 \mathrm{~mm}, 24 \mathrm{~mm}$, and $37.5 \mathrm{~mm}$ deep about the $12 \mathrm{~mm}, 16 \mathrm{~mm}$, and $25 \mathrm{~mm}$ boulders, respectively, for the lift force to be independent of flow depth.

At the surge front, the flow is about $16 \mathrm{~mm}$ deep (see Fig. 4(a)), nearly satisfying the submergence depth criteria for the $12 \mathrm{~mm}$ boulder, but not for the $16 \mathrm{~mm}$ and $25 \mathrm{~mm}$ boulders. Accordingly, the estimation of lift force we used is not representative. Although the lift force applied to the boulder might be affected by the presence of the water surface, as scour develops about the boulder, the lift force is no longer the control factor for the pickup. It is emphasized that the foregoing explanations for the discrepancy in pickup timings are our conjecture.

Let us recognize that the shear force is negligibly small compared to the pressure force for a highly turbulent flow. According to Archenbach [1972], the ratio of shear force to the total drag decreases as $\approx R^{-0.5}$ : here, $R$ is the Reynolds number. In the present study, the Reynolds number is approximately $10^{5}$, and, based on Achenbach's experimental data, the shear force is less than $2 \%$ of the total drag. For a spherical-shaped object, the applied pressure force must act through the geometric centroid of the sphere. This is true regardless of the force types that are artificially classified as drag, lift, and inertial forces. Consequently, for the partially buried sphere to overturn about its downstream edge, the net force acting on the boulder must point upward above that moment axis. Since the moment axis for the halfburied sphere is at the same elevation as its centroid, the lift force must be greater than the buoyant weight for the net force to cause its rotational movement to pull it up, assuming that the sphere does not displace the surround soil. As scour about the sphere develops, hence the moment axis becomes lower than the elevation of the centroid, the ratio of lift to drag force needed to overturn the boulder is reduced. In this way, flow with a high Shields number (and corresponding scour rate) may impact the boulder transport process. For boulders that are buried more than half way in the soil, the boulder may not rotate from initial location without displacing the surrounding soil and the lift force must be greater than the sum of the buoyant weight of the sphere plus the overburden soil to initiate transport. The foregoing discussion suggests that a new form of the Shield number may be worth defining for the boulder pickup problem: namely the ratio of the lift force to the buoyant weight,

$$
\frac{C_{s} u_{0}^{2}}{g D\left(G_{s}-1\right)},
$$

where $C_{s}$ represents the amount of boulder surface exposed to the flow and $D$ represents a length scale of the boulder.

In Trial Two with three $16 \mathrm{~mm}$ boulders buried one-quarter, one-half, and threequarters diameter in the soil, only the one-quarter and one-half buried boulders were transported; the three-quarter buried boulder remained in place. The initial burial depth of three-quarters must prevent the spherical-shaped boulder from transport, even when partially exposed to flow conditions that would otherwise initiate transport. It is worth noting that Truelsen et al. [2005] reported that when the burial depths were greater than 0.80 of the sphere's diameter, then no scour occurred. 
Unlike the one-quarter and one-half buried cases in our experiments, the threequarter buried sphere resisted dislodgement.

\section{Summary and Conclusion}

In summary, the traditional approach to determine the incipient movement of boulder pickup are adapted for the case of a half buried spherical boulder. Predicted and measured movement of the boulders is compared. Based on the experiments, we found that the inertial forces are not responsible for initiating boulder pickup: the timing of incipient movement is clearly after the subsidence of the inertial force that peaks at the initial impact of the surge. For a spherical-shaped boulder, the net force (neglecting friction) must be exerted through the geometric centroid. Hence, when a homogeneous boulder is at least one-half buried in the soil, the only mechanism that causes the rotational motion is the net upward force (i.e. lift force). Our experimental results show that timing of the transport process cannot be predicted by the traditional approach: force and moment balance by a linear combination of drag, lift, inertial, and body forces. To explain the difference, two possible physical factors are discussed: pore-water pressure dissipation in the soil, and the influence of the water surface on the fluid forces. Pore-water pressure dissipation process in the soil would increase the stabilizing body force and create a delay in transport of the boulder: this is a conjecture because pore-water pressure beneath the sphere are not measured in the experiments. Our simplified evaluation suggests that the presence of a shallow water surface - the depth being comparable to the boulder dimension - could have an impact on the fluid forces applied to the boulders.

\section{Acknowledgments}

The authors thank Ben Mason and Bruce Kutter for their valuable advice and support for preparation of the experiments. The experiments were conducted at the Center for Geotechnical Modeling at the University of California, Davis, with support of the National Science Foundation (CMMI-1538211). HY acknowledges the National University of Singapore for his travel support.

\section{References}

Achenbach, E. [1972] "Experiments on the flow past spheres at very high Reynolds numbers," J. Fluid Mech. 54(3), 565-575.

Alexander, J. and Cooker, M. J. [2016] "Moving boulders in flash floods and estimating flow conditions using boulders in ancient deposits," Sedimentology 63(6), 1582-1595.

Bung, D. B. and Valero, D. [2016] "Optical flow estimation in aerated flows," J. Hydraul. Res. $\mathbf{5 4}(5), 575-580$.

Dean, R. G. and Dalrymple, R. A. [1991] Water Wave Mechanics for Engineers and Scientists (World Scientific Publishing Company, Singapore).

Farnebäck, G. [2003] "Two-frame motion estimation based on polynomial expansion," Scandinavian Conf. Image Analysis, Halmstad, Sweden, pp. 363-370. 
Imamura, F., Goto, K. and Ohkubo, S. [2008] "A numerical model for the transport of a boulder by tsunami," J. Geophys. Res. Oceans $\mathbf{1 1 3}(\mathrm{C} 1), 12$ p.

Kennedy, A. B., Mori, N., Zhang, Y., Yasuda, T., Chen, S., Tajima, Y., Pecor, W. and Toride, K. [2016] "Observations and modeling of coastal boulder transport and loading during Super Typhoon Haiyan," Coastal Eng. J. 58(1), 1640004-1-1640004-25.

Koshimura, S. and Hayashi, S. [2012] "Tsunami flow measurement using the video recorded during the 2011 Tohoku tsunami attack," Geoscience and Remote Sensing Symp. (IGARSS), 2012 IEEE Int., Munich, Germany, pp. 6693-6696.

Lamb, H. [1932] Hydrodynamics (Cambridge University Press, Cambridge).

Liu, H., Sakashita, T. and Sato, S. [2014] "An experimental study on the tsunami boulder movement," Coastal Eng. Proc. 1(34), 16.

Luccio, P. A., Voropayev, S. I., Fernando, H. J. S., Boyer, D. L. and Houston, W. N. [1998]. "The motion of cobbles in the swash zone on an impermeable slope," Coastal Eng. 33(1), $41-60$.

Nandasena, N. A. K., Paris, R. and Tanaka, N. [2011] "Reassessment of hydrodynamic equations: Minimum flow velocity to initiate boulder transport by high energy events (storms, tsunamis)," Marine Geology 281(1-4), 70-84.

Nandasena, N. A. K. and Tanaka, N. [2013] "Boulder transport by high energy: Numerical model-fitting experimental observations," Ocean Eng. 57, 163-179.

Noji, M., Imamura, F. and Shuto, N. [1993] "Numerical simulation of movement of large rocks transported by tsunamis," Proc. IUGG/IOC Int. Tsunami Symp., Wakayama, Japan, pp. 189-197.

Noormets, R., Crook, K. A. W. and Felton, E. A. [2004] "Sedimentology of rocky shorelines: 3.: hydrodynamics of megaclast emplacement and transport on a shore platform, Oahu, Hawaii," Sedimentary Geology 172(1-2), 41-65.

Nott, J. [2003] "Waves, coastal boulder deposits and the importance of the pre-transport setting," Earth Planet. Sci. Lett. 210(1-2), 269-276.

Petroff, C. M., Moore, A. L. and Arnason, H. [2001] "Particle advection by turbulent boresorientation effects," Proc. Int. Tsunami Symp, Seattle, Washington, pp. 897-904.

Prandtl, L. and Tietjens, O. [1934] Applied Hydro- and Aerodynamics (Dover Publications, Inc.).

Stephenson, W. J. and Abazović, A. [2016] "Measuring coastal boulder movement under waves using tri-axial accelerometers," J. Coast. Res. 75(sp1), 607-611.

Truelsen, C., Sumer, B. M. and Fredsøe, J. [2005] "Scour around spherical bodies and selfburial," J. Waterw. Port, Coast. Ocean Eng. 131(1), 1-13.

Whitham, G. B. [1955] "The effects of hydraulic resistance in the dam-break problem," Proc. R. Soc. Lond. Ser. A. Math. Phys. Sci. 227(1170), 399-407.

Yeh, H. and Mason, H. B. [2014] "Sediment response to tsunami loading: Mechanisms and estimates," Geotechnique 64(2), 131-143. 